\title{
Shuffle vs. Kautz/De Bruijn Logical Topologies for Multihop Networks: a Throughput Comparison
}

\author{
F. Bernabei *, V. De Simone *, L. Gratta *, M. Listanti \# \\ * Fondazione Ugo Bordoni - via B. Castiglione 59, 00142 Roma (Italy); \\ e-mail:\{bernabei, laura\}@fub.it \\ \# INFOCOM Dept., University "La Sapienza"; via Eudossiana 18, 00184 Roma \\ (Italy); e-mail: marco@infocom.ing.uniroma1.it
}

\begin{abstract}
This paper deals with the analysis of the throughput performance of various logical topologies for Multihop Networks. In particular, ShuffleNets, De Bruijn graphs and Kautz graphs are analyzed. For the comparison, routing algorithms adopting minimum path length are considered. A hot-spot traffic scenario is adopted, modeling the presence of a centralized network resource to which a quota of the internal traffic is directed or originated from. The analysis is carried out by varying the traffic unbalance degree, from a uniform traffic distribution to a completely unbalanced one (all the traffic is concentrated in the hot-spot node).

For ShuffleNets, simple analytical expressions of the actual throughput limits are utilized. In the case of De Bruijn and Kautz graphs, instead, a lower bound of the throughput is utilized, which coincides with the actual throughput in a wide range of values of the network size.

The results obtained show that Shuffle and Kautz graphs always outperform De Bruijn topologies. Moreover, ShuffleNets present a further advantage on the other topologies; in fact, since the nodes are topologically equivalent, the placement of the hot-spot node does not affect the throughput performance.
\end{abstract}

\section{Keywords}

Multihop lightwave networks, Shuffle Networks, De Bruijn graphs, Kautz graphs

\section{INTRODUCTION.}

Multihop Networks (MNs) [1,2] represent an attractive solution to implement optical infrastructure interconnecting a large set of stations distributed in a local-metropolitan area.

In MNs the physical topology must be distinguished from the logical topology. The former consists in the physical medium connecting the network nodes, and is usually a star or bus; the latter is overlaid on the physical one, for example using a number of different wavelengths each between a pair of stations. In this way, each node is connected, by means of dedicated logical links, to a set of other nodes; so, a packet must be forwarded for multiple hops to reach its destination if origin and destination nodes are not directly connected in the logical topology. 
The logical topology can be usefully represented by means of a graph. The indegree and the outdegree of the graph are represented by the number of logical links incoming to and outgoing from a node, respectively. The graph diameter determines the minimum number of hops needed, in the worst case, to go from to a node to another. In the class of graphs with $N$ nodes and maximum outdegree $p$ the minimum diameter is of $O\left(\log _{p} N\right)$. A logical topology should be designed aiming at: i) simplifying the routing algorithm; ii) reducing, as much as possible, the number of hops to reach a destination node; iii) avoiding bottlenecks in the network in order to increase the maximum throughput. In this paper, three logical topologies are described (Shuffle, Kautz and De Bruijn topologies) and their throughput performance are compared.

The Shuffle logical topology is based on Perfect Shuffle graphs. Many papers dealt with ShuffleNets (SNs) $[1,3,4,5,6,7]$. They showed the highly efficient use of the communication channel under uniform traffic, whereas they outlined a remarkable throughput decrease in case of non-uniform load, partially limited by the use of an adaptive packet routing scheme.

Logical topologies based on De Bruijn and Kautz graphs have been proposed and studied in recent papers $[8,9]$. The comparison with the SNs is basically focused on topological properties and is limited to a uniform traffic scenario.

In [8] it is stated that, for the same maximum degree and average number of hops, logical topologies based on De Bruijn graphs can support a much larger number of nodes than ShuffleNets. Networks based on De Bruijn graphs have higher throughput and lower average delay. In [9] a comparison between De Bruijn and Kautz graphs is discussed. The main outcomes are that logical topologies based on Kautz graphs can accommodate a greater number of nodes than that given by a De Bruijn graph with the same degree and diameter. Moreover, the average queueing delay in a Kautz graph network gives better results than in a network based on De Bruijn graphs.

In this paper, a more complete analysis of the throughput performance of the three topologies is carried out, with reference to modulated hot-spot traffic configurations. Over a background given by a uniform traffic component, a hot spot traffic models the presence of a centralized network resource (e.g. a file server, a mass storage equipment, etc.) which a quota of the internal traffic is directed to or is originated from. As a particular case, if the hot spot component vanishes, a uniform traffic configuration is obtained. A high hot-spot traffic quota leads to critical conditions for MN performance, since it involves high loads on links incoming to and outgoing from the hot spot. So, a worsening of throughput performance is expected with respect to the uniform case. The study aims at evaluating the capacity of various logical topologies to mitigate the throughput degradation as the hot spot traffic quota increases.

Simple analytical expressions of the actual throughput limits, derived in [7], are used for Shuffle Networks, whereas for the other topologies an enumeration approach is applied.

Obtained results show that the performance of all the three topologies are strongly influenced by the traffic unbalance degree. Moreover, De Bruijn graphs always perform worst than Kautz graphs and ShuffleNets. However, for high values of traffic unbalance and network size the differences among the three topologies tend to vanish, and they fundamentally depend only on the network size and on the traffic unbalance value. Lastly, ShuffleNets have the exclusive advantage that, since all nodes are topologically equivalent, there is no impact of the hot-spot node placement on the throughput performance.

The paper is organized as follows. In Sec. 2 the logical topologies are described; in Sec. 3, the adopted traffic configuration is explained. In Sec. 4 the procedures employed for the performance evaluation are reported, while in Sec. 5 a performance comparison is carried out. 


\section{LOGICAL TOPOLOGIES FOR MULTIHOP NETWORKS}

In this section, Shuffle, Kautz and De Bruijn logical topologies are described. Let $\mathcal{N}$ and $\mathcal{L}$ be the set of nodes and the set of links of a generic MN, respectively. Any node is able to detect $p$ input frequencies and to transmit over $p$ output frequencies. Whichever the logical network topology is, it results: $|\mathcal{L}|=p|\mathcal{V}|$, wherein $|\mathcal{S}|$ denotes the cardinality of the set $\mathcal{S}$.

\subsection{Shuffle topology}

A $(p, k)$ ShuffleNet (SN) [2] is composed of $|\mathcal{K}|=N^{S}=k p^{k}$ nodes, logically arranged in $k$ columns. Two adjacent columns are connected by means of a $p$-shuffle; the $k$ th column is connected to the first one, in such a manner that the overall connectivity graph is wrapped around a cylinder.

A generic node $\chi \in \mathcal{N}$ is identified by the couple $\left[c_{\mathbf{x}}, \mathbf{x}\right]$, where $c_{\mathbf{x}}\left(0 \leq c_{\mathbf{x}} \leq k-1\right)$ is the column index and $\mathbf{x}$ represents the row index, i.e. the string $\left\langle\mathrm{x}_{\mathbf{k}}, \mathrm{x}_{\mathrm{k}-1}, \ldots, \mathrm{x}_{1}\right\rangle$ being the $p$-ary representation of the row index of the node $x$. Moreover, $\left[c_{\mathbf{x}}, \mathbf{x}, \mathbf{x}_{0}\right]$ represents the node $\chi$ outgoing link, identified by the routing digit $\mathrm{x}_{0}$ processed by the node itself. Each value of the digit $\mathrm{x}_{0}$ corresponds to a specific output link. The node $x=\left[c_{\mathbf{x}},\left\langle\mathbf{x}_{\mathbf{k}}, \mathrm{x}_{\mathrm{k}-1}, \ldots, \mathrm{x}_{1}>\right]\right.$ is connected to the $p$ nodes $\left[\mathrm{c}_{\mathrm{x}+1},<\mathrm{x}_{\mathrm{k}-1}, \mathrm{x}_{\mathrm{k}-2}, \ldots, \mathrm{x}_{1}, \mathrm{x}_{0}>\right]$ obtained assigning the $p$ possible values to $\mathrm{x}_{0}$. Figure 1 shows the logical topology of a $(2,2)$ SN.

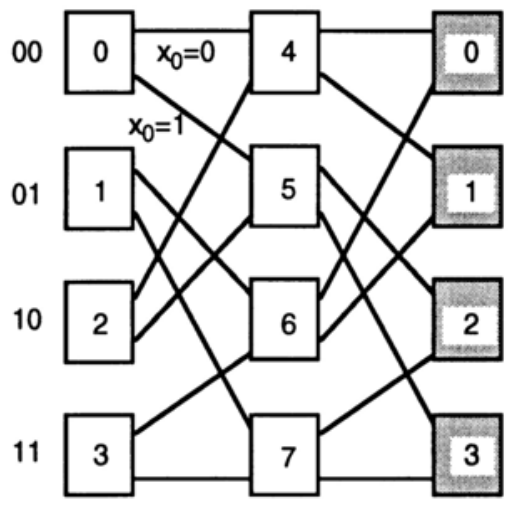

Figure $1(2,2)$ Shuffle logical topology.

\subsection{Kautz topology}

A $(p, k)$ Kautz Network $(\mathrm{KN})[9]$ is composed of $|\mathcal{K}|=N^{K}=p^{k}+p^{k-1}$. The generic node $\chi \in \mathcal{N}$ is identified by a string $\mathbf{X}=<\mathbf{x}_{\mathbf{k}}, \mathbf{x}_{\mathbf{k}-1}, \ldots, \mathrm{x}_{1}>$ of $k(p+1)$-ary digits, called node index. Each link is identified by the couple $\left[\mathbf{x}, \mathrm{x}_{0}\right]$, being $\mathrm{x}_{0}$ the routing digit processed by the node itself. The Figure 2 represents a $(2,2) \mathrm{KN}$. The generic node $\left\langle\mathrm{x}_{\mathbf{k}}, \mathrm{x}_{\mathbf{k}-1}, \ldots, \mathrm{x}_{1}\right\rangle$ is connected to the $p$ nodes $\left\langle\mathrm{x}_{\mathrm{k}-1}, \mathrm{x}_{\mathrm{k}-2}, \ldots, \mathrm{x}_{1}, \mathrm{x}_{0}\right\rangle$ obtained by assigning the $p$ possible values to $\mathrm{x}_{0}$ such that $\mathrm{x}_{0} \neq \mathrm{x}_{1}$. It is to be noted that this constraint implies that there is no link connecting a node to itself, and there is no node such that: $x_{i}=x_{i+1}, \forall i \in[0, k-1]$.

\subsection{De Bruijn topology.}

A $(p, k)$ De Bruijn Network (DN) [8] is composed of $|\mathcal{K}|=N^{D}=p^{k}$. The node index of a generic node $x \in \mathcal{N}$ is identified by a string $\mathbf{x}=<\mathbf{x}_{\mathbf{k}}, \mathbf{x}_{\mathbf{k}-1}, \ldots, \mathbf{x}_{1}>$ of $k p$-ary digits. Each link is identified by the couple $\left[\mathbf{x}, \mathrm{x}_{0}\right]$, being $\mathrm{x}_{0}$ the routing digit processed by the node itself. The Figure 3 represents a $(2,3)$ DN. The generic node $\left\langle x_{k}, x_{k-1}, \ldots, x_{1}\right\rangle$ is connected to the $p$ nodes $\left\langle\mathbf{x}_{\mathbf{k}-1}, \mathbf{x}_{\mathbf{k}-2}, \ldots, \mathbf{x}_{1}, \mathrm{x}_{0}\right\rangle$ obtained by assigning the $p$ possible values to $\mathbf{x}_{0}$. It is to be noted that, if all the digits of the node index are equal, there is one link connecting the node to 
itself. As it will be shown in the following, the presence of these links impairs the throughput performance of DNs as compared to KNs.

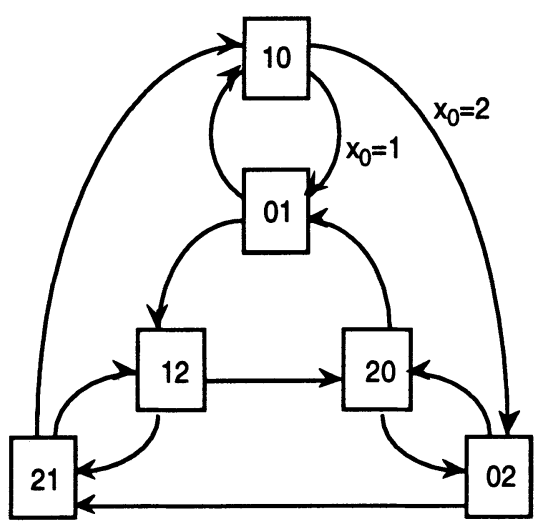

Figure $2(2,2)$ Kautz logical topology.

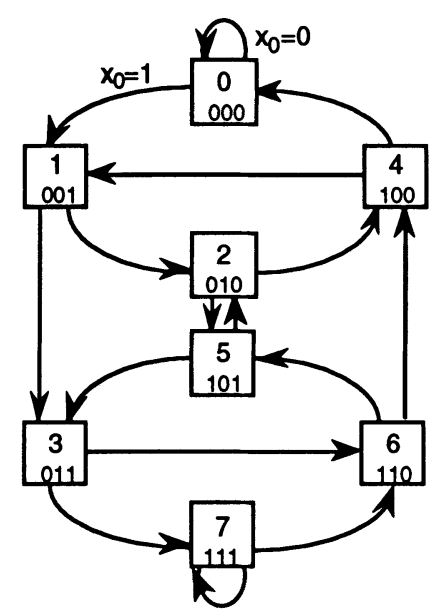

Figure 3 (2,3) De Bruijn logical topology.

\section{TRAFFIC SCENARIO.}

In this section, the adopted traffic scenario is described, and the performance measures used in the comparison of the logical topologies are introduced. In the following, all the traffic measures are intended as normalized with respect to the link capacity, supposed equal for all the network links. Let:

$a_{0 x y}$ the normalized mean traffic generated by the traffic relation $(x, y)$; it is worth noting that, the traffic components relevant to the traffic relations originating from and terminating at the same node are not considered, therefore $a_{o x x}=0, \forall x$;

$a_{c x y}$ the normalized mean carried traffic relevant to the traffic relation $(x, y)$;

$A_{o x}$ the overall normalized mean traffic offered by a node $\chi \in \mathcal{N}$ i.e. $A_{O X}=\Sigma_{y \in \mathcal{X}} a_{0 x y}$;

$A_{\mathrm{cx}}$ the normalized throughput of a node $\chi \in \mathcal{N}$ defined as the sum of the normalized mean carried traffic relevant to all the traffic relations originating from the node i.e.

$A_{\mathrm{cx}}=\Sigma_{y \in \mathcal{X}} a_{\mathrm{cxy}}$;

$A_{0}$ be the overall normalized mean load offered to the network, i.e. $A_{0}=\Sigma_{\chi \in \mathcal{K}} A_{o \chi}$;

$A_{\mathrm{c}}$ be the normalized total traffic carried by the network, i.e. $A_{\mathrm{c}}=\Sigma_{\chi \in \mathcal{X}} A_{\mathrm{C} \chi}$.

The evaluation of the maximum network throughput is here carried out by considering traffic configurations resulting from the superposition of different percentages of uniform and hot-spot traffic patterns. A specific traffic configuration is characterized by: a) a percentage, $\alpha,(0 \leq \alpha \leq 1)$, called hot-spot factor, of $A_{0}$ associated with the traffic relations originating from and destined to a given node (that is, the hot-spot); b) the remaining load, $(1-\alpha) A_{0}$, is uniformly distributed among all the traffic relations.

Let the node $z$ be the hot-spot; by considering that the number of traffic relations originating 
from or terminating at $z$ is equal to $2 \cdot(N-1)$, we have:

$$
a_{0 x y}(\alpha)=\left\{\begin{array}{ll}
\frac{F}{N-1} & x \neq z \text { and } y \neq z \\
\frac{F}{N-1}+\frac{\alpha A_{O}}{2(N-1)} & x=z \text { or } y=z
\end{array} \quad A_{0 x}(\alpha)= \begin{cases}F+\frac{\alpha A_{0}}{2(N-1)} & x \neq z \\
F+\frac{\alpha A_{0}}{2} & x=z\end{cases}\right.
$$

wherein $F=\frac{(1-\alpha) A_{0}}{N}$.

It is to be noted that, $\alpha=0$ corresponds to the uniform traffic case. In the following, for the sake of brevity, a traffic configuration with $\alpha \neq 0$ will be called " $\alpha$-hot-spot".

The $\alpha$-hot-spot traffic configuration models the presence of a centralized network resource, corresponding to the hot-spot node (e.g. a file server, a mass storage equipment, etc.), to which a quota of the internal traffic is directed or is originated from. The higher $\alpha$ is, the more the traffic configuration represents a critical case. This is due to the fact that it involves high loads on incoming and outgoing hot-spot node links. Therefore, a worsening of throughput performance can be expected as $\alpha$ increases.

If a value of $\alpha$ is fixed, the normalized maximum throughput, $T_{\max }(\alpha)$, is defined as the maximum value of traffic carried by the network (normalized with respect to the number of nodes $N$ ) provided that the characteristics of the carried traffic be identical with those of the offered one, i.e.

$T_{\max }(\alpha)=\left\{\frac{1}{N} \max _{\mathrm{A}_{0}}\left[A_{\mathrm{c}}\right] \mid \frac{a_{\mathrm{cxy}}}{a_{\mathrm{cz} w}}=\frac{a_{0 x y}}{a_{0 z w}}, \forall x, y, z, w\right\}$

In the case of uniform load, $T_{\max }(\alpha=0)$ represents the maximum value of the offered traffic such that the traffic carried by the network be equally distributed over all the traffic relations.

It is worth noting that in some cases $T_{\max }(\alpha)$ does not correspond to the actual maximum possible value of network throughput. In fact, if the network links are not uniformly loaded, a node could generate, at least in principle, more traffic over those paths crossing non-congested network zones. That would lead to a higher throughput, but the distribution of the carried traffic on the various traffic relations would not correspond to the hypothesized characteristics of the offered traffic. In this sense, $T_{\max }(\alpha)$ corresponds to the maximum throughput arising from a perfectly "fair" network operation with respect to the various traffic relations.

According to the hypotheses on the traffic model, the evaluation of $T_{\max }(\alpha)$ can be carried out by identifying the link carrying the maximum load and by counting the number of paths belonging to the uniform and hot-spot traffic components crossing that link.

Let $l \in \mathcal{L}$ be a generic network link. Let:

$N^{x} \quad$ the number of nodes of a generic topology $(x=\mathrm{S}, \mathrm{K}, \mathrm{D})$;

$N_{h}(l)$ the number of paths belonging to the hot-spot traffic component passing through $l$;

$N_{u}(l)$ the number of paths belonging to the uniform traffic component passing through $l$;

$l_{\max }$ the most loaded link(s) in the network;

$a(\ell)$ the mean traffic carried by the link $l$.

It results:

$a\left(\zeta_{\max }\right)=\max _{l \in \mathcal{L}}\left\{N_{u}(\iota) \frac{(1-\alpha) A_{0}}{N^{x}\left(N^{\mathrm{x}}-1\right)}+N_{h}(\Omega) \frac{\alpha A_{0}}{2\left(N^{x}-1\right)}\right\}$

Since $a\left(\zeta_{\max }\right) \leq 1$, and normalizing with respect to $N^{x}$, it follows that:

$T_{\max }^{x}(\alpha)=\frac{2\left(N^{x}-1\right)}{2(1-\alpha) N_{u}\left(l_{\max }\right)+\alpha N^{x} N_{h}\left(C_{\max }\right)}$ 


\section{MAXIMUM THROUGHPUT EVALUATION.}

A sequence of links and nodes connecting two nodes $x$ and $y$ defines a path. The number of hops of a path identifies the path length. In order to identify a path of length $h$ a string of $k+h$ digits can be used. A schematic description of the general path format is given in Figure 4. The first $k$ digits correspond to the index (row index for SNs, node index for KNs and DNs) of

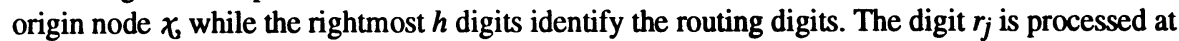
the $j^{\text {th }}$ hop. A rightward sliding window of width $k$ identifies, step by step, the index of all the crossed nodes from the origin to the destination. In particular, the last $k$ digits identify the index of the destination node $y$. Routing algorithms for Multihop Networks can be classified according to the criterion adopted to fix the number and the value of the routing digits $\left\langle\mathrm{r}_{1}, \mathrm{r}_{2}, \ldots, \mathrm{r}_{\mathrm{h}}\right\rangle$.

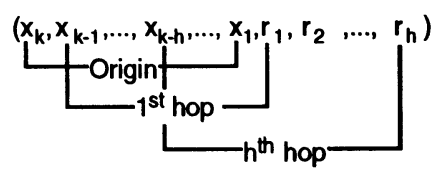

Figure 4 General path format.

The performance comparison of the three logical topologies is carried out by uniquely considering minimum path length algorithms. In order to better understand the comparison, it is to be noted that, in the case of SNs, since the minimum length path is not unique, the multipath capability can be exploited. This does not hold for Kautz and De Bruijn, since there is a single minimum length path.

In the following, a brief description of the minimum length routing algorithms adopted for the three logical topologies will be given. At this aim, we give some definitions.

Let $\mathbf{x}=\left\langle\mathbf{x}_{\mathbf{k}}, \mathbf{x}_{\mathbf{k}-1}, \ldots, \mathrm{x}_{1}\right\rangle$ be a string of $k$ digits. We denote as $\lambda_{\mathrm{i}}(\mathbf{x}), i \leq k$, the $i$ least significant digits of $\mathbf{x}$, i.e., $\lambda_{\mathrm{i}}(\mathbf{x})=\left\langle\mathrm{x}_{\mathrm{i}}, \mathrm{x}_{\mathrm{i}-1}, \ldots, \mathrm{x}_{1}\right\rangle$ and with $\mu_{\mathrm{i}}(\mathrm{x}), i \leq k$, the $i$ most significant digits of $\mathbf{x}$, i.e., $\mu_{\mathrm{i}}(\mathbf{x})=\left\langle\mathbf{x}_{\mathbf{k}}, \mathbf{x}_{\mathbf{k}-1}, \ldots, \mathbf{x}_{\mathbf{k}-\mathrm{i}+1}\right\rangle$. Moreover, $\delta_{\mathbf{k}}$ represents a string composed of $k$ don't care digit. Lastly, $\mathbf{x} \cdot \mathbf{y}$ represents the juxtaposition of the two strings $\mathbf{x}$ and $y$.

\subsection{Maximum throughput in SNs}

Let $w$ be the number of columns from the origin to the destination node; it results:

$$
w= \begin{cases}\left(k+c_{\mathrm{y}}-c_{\mathrm{x}}\right) \bmod k & \text { if } c_{\mathrm{y}} \neq c_{\mathrm{x}} \\ k & \text { if } c_{\mathrm{y}}=c_{\mathrm{x}}\end{cases}
$$

wherein $c_{\mathrm{x}}$ and $c_{\mathrm{y}}$ are the column indexes of nodes $x$ and $y$, respectively.

The minimum path length is equal to either $w$ or $w+k$, and therefore its range is between 1 and $2 k-1$. Let $\mathcal{M}(x, y)$ be the set of minimum length paths for the traffic relation $(x, y)$. If $h=w$ the minimum length path is unique $(|\mathcal{M}(x, y)|=1)$ and is unambiguously determined by the string $\mathbf{x} \cdot \lambda_{w}(y)$, otherwise there exist $p^{w}$ minimum length paths $\left(|\mathcal{M}(x, y)|=p^{w}\right)$, which are obtained by fixing arbitrarily the first $w$ routing digits of the path $\mathbf{x} \cdot \delta_{w} \cdot y$.

In [2] a fixed minimum length routing algorithm, called in the following Fixed Routing on ShuffleNets (FR), is proposed. According to this algorithm, if $\mu_{\mathbf{k}-w}(\mathbf{y})=\lambda_{\mathbf{k}-\mathrm{w}}(\mathbf{x})$ the routing path is $\mathbf{x} \cdot \lambda_{w}(y)$; otherwise it is $\mathbf{x} \cdot \lambda_{w}(y) \cdot y$. This algorithm identifies, for each traffic relation, a unique route, and therefore it does not exploit the multipath capability of SNs. More details on the properties of FR can be found in [7].

Closed formulae for the evaluation of the maximum throughput for FR $\left(T_{\max }^{F R}(\alpha)\right)$ are 
demonstrated in [7]; here we only recall the fundamental results of that analysis. Given a $(p, k)$ SN operating according to FR and loaded by an $\alpha$-hot spot traffic, with $0 \leq \alpha \leq 1, N_{h}\left(L_{\max }\right)$ and $N_{u}\left(\zeta_{\max }\right)$, are given by:

$\left\{\begin{array}{lll}N_{h}\left(\zeta_{\max }\right)=4 & N_{u}\left(\mathcal{L}_{\max }\right)=8 & \text { if } k=p=2 \\ N_{h}\left(\mathcal{C}_{\max }\right)=(k-1) p^{\mathrm{k}} & N_{u}\left(\zeta_{\max }\right)=\frac{p^{\mathrm{k}+1}\left[p^{3}\left(k^{2}-k\right)-p\left(3 k^{2}-k\right)+2 k^{2}\right]-2 k+2 k p}{2 \cdot(1-p)^{2}} & \text { otherwise }\end{array}\right.$

Therefore $T_{\max }^{F R}(\alpha)$ can be evaluated by substituting eq.(3) in eq.(2).

Throughput advantages can be obtained if the multipath capability is exploited, still keeping the minimum length constraint. The maximum throughput for minimum path length algorithms can be found by solving a linear optimization problem (Globally Optimized Minimum length Routing, GOMR). For network sizes greater than a few tens of nodes, the solution of the problem becomes unfeasible. However, a tight upper bound for the maximum network throughput $\left(U_{G O M R}(\alpha)\right)$ is given by [7]:

$U_{G O M R}(\alpha)=\frac{1}{\max \left[C_{1}(k, \alpha), C_{2}(k, \alpha)\right]}$

where

$C_{1}(k, \alpha)=\frac{\alpha N(N-1)(1-p)^{2}+2(1-\alpha)\left[k\left(p^{\mathbf{k}+2}-p^{\mathrm{k}+1}-(1-p)^{2}\right)+p^{2}-p^{\mathrm{k}+2}+(1-p)^{2} k p^{\mathbf{k}}\right]}{2 p(1-p)^{2}(N-1)}$

$C_{2}(k, \alpha)=\frac{3 k^{2} p^{\mathrm{k}}(1-p)+k p^{\mathrm{k}}(1+p)-2 k}{2 p(1-p)(N-1)}$

The upper bound provided by eq. (4) is very close to the actual value of the maximum throughput, except for low values of $\alpha$ for the $(2,2)$ SN. For any other values of $p$ and $k$ the upper bound practically coincides with the maximum, as it arises from the analysis of Figure 5.

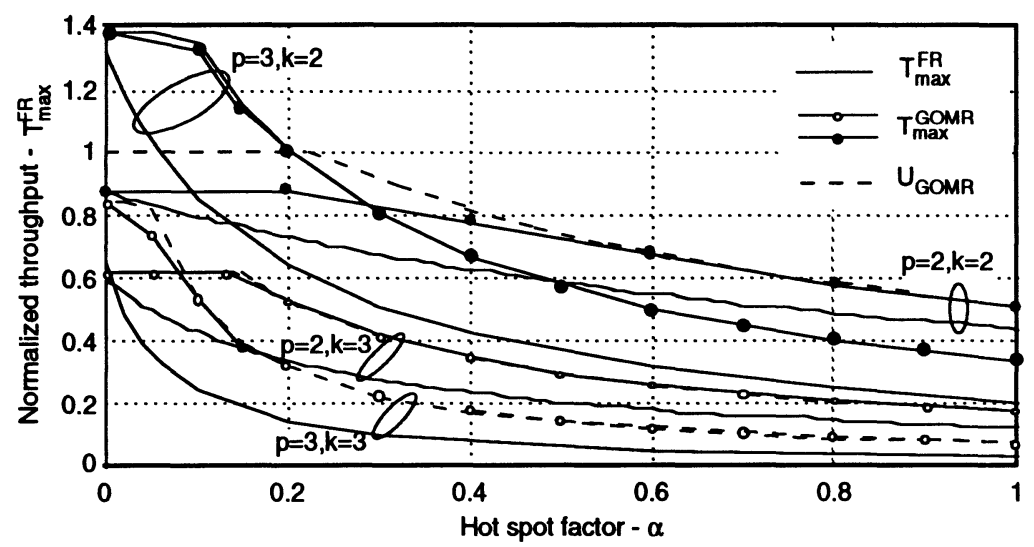

Figure 5 Throughput limits for ShuffleNets, $p=2$ and 3.

This figure plots $T_{\max }^{F R}(\alpha), T_{\max }^{G O M R}(\alpha)$ and $U_{G O M R}(\alpha)$ vs. $\alpha$ for $k=2,3$, for $p=2$ and 3, respectively. In the following, $U_{G O M R}(\alpha)$ will be employed in the performance comparison with DNs and KNs, except in the case of the $(2,2) \mathrm{SN}$, where $T_{\max }^{G O M R}(\alpha)$ will be reported.

From the figure it is evident that the node throughput considerably decreases as $\alpha$ increases; however, for low values of $\alpha$ GOMR is able to achieve a throughput close to the value relevant 
to the uniform traffic case. It must be observed that, in the SN case, the hot-spot node can be placed in any network node without affecting the throughput performance, since all the nodes are topologically equivalent [7]. This is not true for the other network topologies, as it will be shown in the following.

\subsection{Maximum throughput in KNs and DNs}

The routing algorithm for KNs and DNs is based on the same principles; what follows applies to both topologies. Given a $(p, k) \mathrm{KN}$ or $\mathrm{DN}$, for each traffic relation $(x, y)$ there is a unique minimum length path $(|\mathcal{M}(x, y)|=1)$. The maximum number of hops of a minimum length path is equal to $k$; therefore, the length $s$ of the string representing the path is such that $k+1<s<2 k$. The minimum length path can be found according to the Minimum Length routing on Kautz graphs (MLK) (Minimum Length routing on De Bruijn graphs (MLD)) consisting in the following two steps:

For each traffic relation $(x, y)$,

- find the largest integer $0 \leq i \leq k$ such that $\lambda_{\mathrm{i}}(\mathbf{x})=\mu_{\mathrm{i}}(\mathbf{y})$

- the path from $X$ to $Y$ is identified by $\mathbf{x} \cdot \lambda_{\mathrm{k}-\mathrm{i}}(\mathbf{y})$.

It is to be noted that, taking into account the routing algorithm operation, all the links (nodes) whose index can be obtained by a transliteration of a same link (node) string are topologically equivalent (1). This means that, in the case of links, they are loaded by the same number of paths while, for the nodes, this means that they have the same configuration of incoming and outgoing links.

A detailed description of the above routing algorithm for KNs and DNs and its properties can be found in [9] and [8], respectively.

In order to evaluate the throughput performance of MLK (MLD), $N_{u}\left(\mathcal{L}_{\max }\right)$ and $N_{h}\left(\mathcal{L}_{\max }\right)$ must be evaluated. That would imply the count of the number of paths crossing each network link.

As for the uniform component, a useful upper bound for $N_{u}\left(f_{\max }\right)$, that holds for both topologies, can be easily found. The generic path passing through $l=\left\langle\mathrm{l}_{\mathrm{k}}, \mathrm{l}_{\mathrm{k}-1}, \ldots, \mathrm{l}_{1}, \mathrm{l}_{0}\right\rangle$ can be written as $\delta_{\mathrm{a}} \cdot\left[\delta_{\mathrm{b}}\right.$, wherein $0 \leq a+b \leq k-1$. All the possible origin nodes $\mathcal{A}$ of the path are such that $\mathcal{A}=\delta_{\mathrm{a}} \cdot \mu_{\mathrm{k}-\mathrm{a}}(\mathcal{l})$, while the possible destination nodes $\mathcal{B}$ are such that $\mathcal{B}=\lambda_{\mathrm{k}-\mathrm{b}}(\mathcal{l}) \cdot \delta_{\mathrm{b}}$. An upper bound of the maximum number of paths passing through $\forall \in \in \mathcal{L}$ is obtained by assigning all the possible values to $\delta_{\mathrm{a}}$ and $\delta_{\mathrm{b}}$, i.e.

$N_{\mathrm{UP}}=\sum_{\mathrm{a}=0}^{\mathrm{k}-1} \sum_{\mathrm{b}=0}^{\mathrm{k}-1-\mathrm{a}} p^{(\mathrm{a}+\mathrm{b})}=\frac{k p^{\mathrm{k}}(p-1)-p^{\mathrm{k}}+1}{(p-1)^{2}}$

$N_{\mathrm{UP}}$ provides an upper bound for the exact number of paths crossing a generic link $L$ In fact, shorter paths connecting $\mathcal{A}$ and $\mathcal{B}$ not passing through $($ could exist. The number of these paths must be taken into account to determine $N_{u}\left(\zeta_{\max }\right)$. However, in most cases $N_{U P}=N_{u}\left(\zeta_{\max }\right)$; this occurs in the following conditions:

- for Kautz topology, if $k \leq 2 p-2$; in fact, there exists a link such that no shorter path can be found among those counted in $\mathrm{N}_{U P}$; in the string relevant to this link at most one digit is repeated, and this digit appears neither in $l_{k}$ nor in $l_{0}$. For example, the string <0121314> (and any its transliteration) represents the most loaded link in a $(4,6) \mathrm{KN}$.

(1) A transliteration of the string $\left\langle\mathbf{x}_{\mathbf{k}}, \mathrm{x}_{\mathbf{k}-1}, \ldots, \mathrm{x}_{1}\right\rangle$ is any string such that at least one digit is substituted, and the equality and inequality relations are kept. For example, in a $(2,2) \mathrm{KN}$ the links $\langle 010\rangle,\langle 101\rangle,\langle 020\rangle$, $<202>,<121>$ and $<212>$ are all transliterations of the same link. 
- for De Bruijn topology, the condition is $k \leq 2 p-4$; in fact, in addition to the above statements, the constraint that two occurrences of the repeated digit cannot be adjacent in the string must be taken into account. This constraint is automatically satisfied in the case of KNs. The string $<0121314>$ represents the most loaded link in a $(5,6) \mathrm{DN}$.

If the above conditions are not satisfied $N_{u}(\ell)$ is here evaluated by enumeration.

As far as $N_{h}(L)$ is concerned, a closed form expression is not presently available; so its evaluation was carried out by enumeration.

Figures 6 and 7 plot the maximum throughput of MLK and MLD vs. $\alpha$, respectively. It is to be noted that, in both cases, the network performance depends on the position of the hot-spot node. In fact, the number of paths crossing the links outgoing from a node varies with the node index, i.e., the network nodes are not topologically equivalent. Consequently, for each value of $\alpha$, there exist a best and a worst position for the hot-spot node; therefore, in the figures the throughput performance is represented by a region delimited by the curves corresponding to the best and worst placement of the hot-spot. It is to be noted that the curves relevant to SN, KN and DN cannot be quantitatively compared, since they are relevant to networks of different size.

Figure 6 plots $T_{\max }^{M L K}(\alpha)$ vs. $\alpha$ for $k=2,3$ and for $p=2,3$. It should be noted that, when $k=2$ (whichever $p$ is), all the node indexes can be derived from the index of a node by means of a transliteration, and therefore they are all topologically equivalent. Consequently, the hot-spot placement has no impact on the throughput.

If $k=3$, in the two cases reported here, the best hot-spot position is the same for any value of $\alpha$, and the throughput decrease caused by a wrong hot-spot placement is not sensitive.

For higher values of $k$ it has been verified that the best hot-spot position varies with $\alpha$, although the relevant curves still have the same qualitative behaviour.

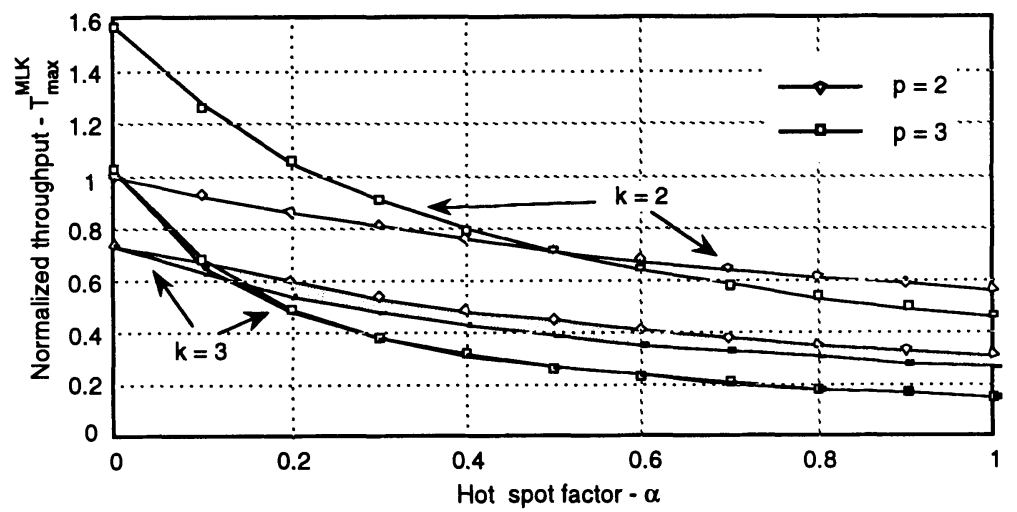

Figure 6 Throughput limits for Kautz graphs, $p=2$ and 3.

Figure 7 plots $T_{\max }^{M L D}(\alpha)$ vs. $\alpha$ for $k=2,3$ and for $p=2,3$. In the DN case, it resulted that in most cases the best hot-spot position varies with $\alpha$.

For example, in the $(2,3)$ case for low values of $\alpha(\alpha \leq 0.3)$ the best position for the hot-spot is in the node $\langle 000\rangle$ (or equivalently in $\langle 111\rangle$ ). That can be justified by considering that the links incoming and outgoing in these nodes are those less loaded by the uniform traffic. Consequently, the addition of the hot-spot traffic component on these links does not cause a more stringent bottleneck to be introduced in the network; conversely, the load on the bottleneck 
links ( $<1001>$ and $<0110>$ ) decreases, since it is mostly determined by the uniform traffic component. This causes a growth of the maximum throughput for low values of $\alpha$. As the traffic unbalance further increases, the best hot-spot position moves to $<010>$ (or $<101>$ ). In fact, since the nodes $\langle 000\rangle$ and $<111\rangle$ have only one incoming and one outgoing link (the recirculating links don't carry traffic) for high values of traffic unbalance these links would become very stringent bottlenecks. The new position of the hot-spot node causes the bottleneck links to be $\langle 0101\rangle$ and $\langle 1010\rangle$ for any further increase of $\alpha$. The same considerations can be carried out for $p=3$. The case $(2,2)$ is somehow degenerate since the uniform traffic component is the same on all the links (excluding $\langle 000\rangle$ and $\langle 111\rangle$ ); consequently, the best hot-spot position is always the same ( $<01>$ or $<10>$ ), and the maximum throughput decreases with $\alpha$. As it can be noted, in the case of DN an inconvenient placement of the hot-spot node can lead to a sensitive worsening of the network performance.

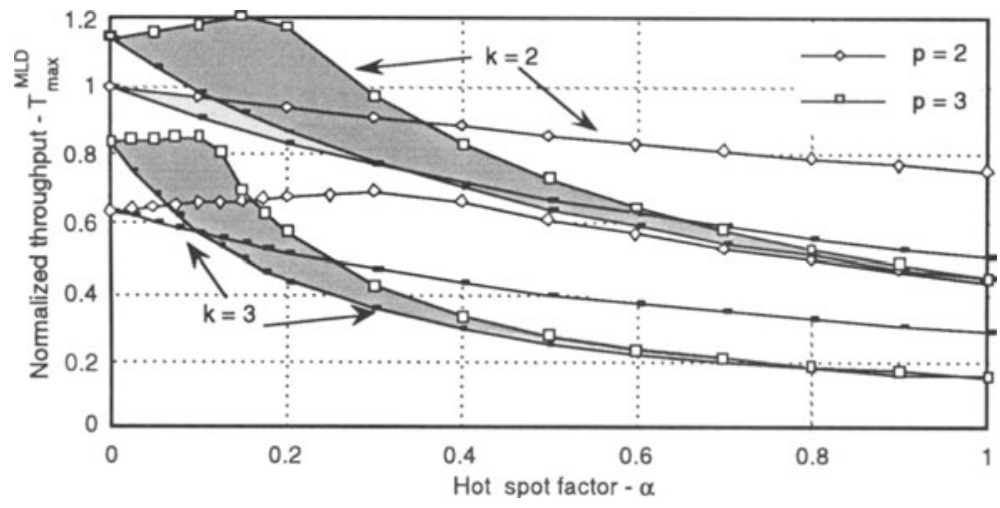

Figure 7 Throughput limits for De Bruijn graphs, $p=2$ and 3.

\section{PERFORMANCE COMPARISON}

In the following, the performance of the three logical topologies, with respect to the maximum throughput they can carry in a hot-spot traffic configuration, will be analyzed.

Figures 8 and 9 plot the maximum normalized throughput vs. the network size $N$ for SN, DN and $\mathrm{KN}$, for $\alpha=0,0.2$ and 1 and for $p=2$ and 4 , respectively. In the case of $S N$, both $T_{\max }^{F R}$ $(\alpha)$ and $U_{G O M R}(\alpha)$ are plotted. The former represents the basic performance of SNs, while the latter represents the best obtainable performance for these networks with minimum length routing algorithms. Although the curve of $U_{G O M R}(\alpha)$ cannot be actually reached unless a globally optimized algorithm is used, the performance of locally optimized adaptive algorithms can be very close to it [7].

If a value of $\alpha \neq 0$ is fixed, the curves relevant to $T_{\max }^{F R}(\alpha)$ for SNs tend toward 0 as $2 / \alpha N$, independently of $p$ [7]. That can be explained by observing that, from eq. (3), $N_{h}\left(f_{\max }\right)$ is equal to $(k-1) p^{\mathrm{k}}$, while the hot-spot component on all the other hot-spot outgoing links is equal to $k p^{k}-1-(k-1) p^{k}=p^{k}-1$. Consequently, the remaining $p$-1 links outgoing from the hot-spot have a negligible effect on the throughput limit. 


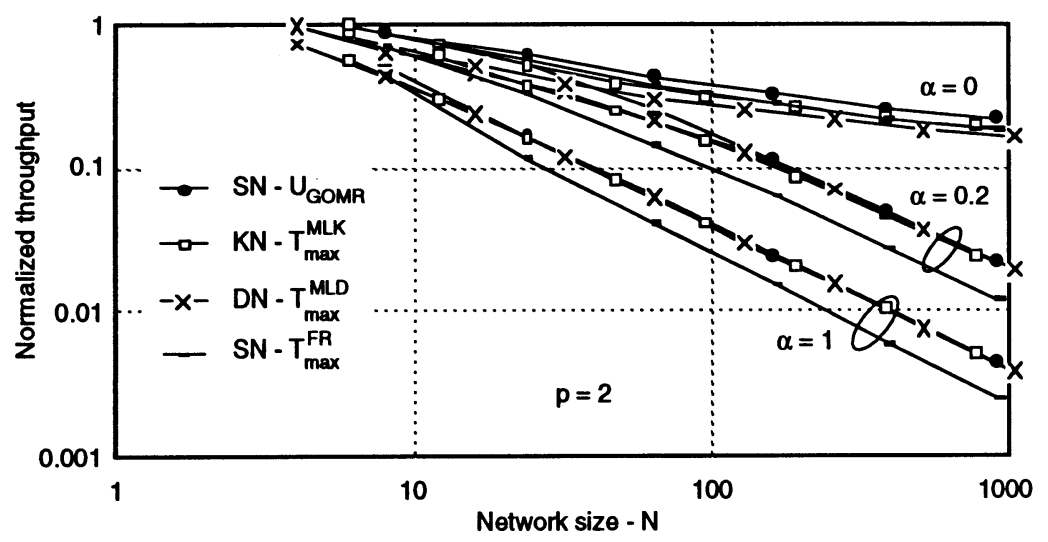

Figure 8 Normalized throughput various values of $\alpha, p=2$.

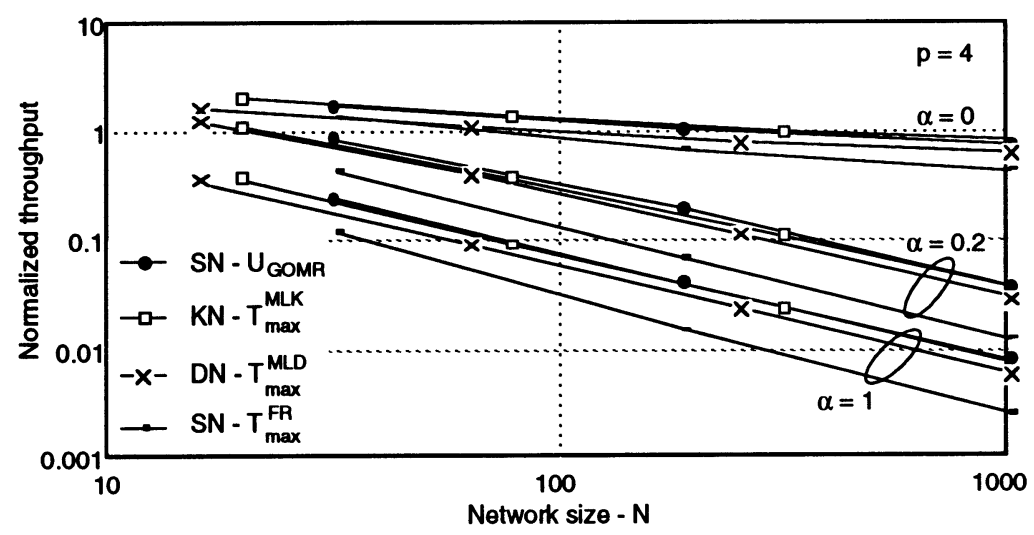

Figure 9 Normalized throughput various values of $\alpha, p=4$.

Conversely, from eq. (4) it results that, when the term $C_{1}(k, \alpha)$ dominates, the curves relevant to $U_{G O M R}(\alpha)$ tend to 0 as $2 p / \alpha N$. Consequently, they are shifted upwards with respect to those relevant to $T_{\max }^{F R}(\alpha)$. By comparing $C_{1}(k, \alpha)$ and $C_{2}(k, \alpha)$ it can be seen that, for increasing values of $k$ and $p, C_{1}(k, \alpha)$ is greater than $C_{2}(k, \alpha)$ even for very small $\alpha$. Conversely, for $\alpha=0$ it is always $C_{1}(k, \alpha)<C_{2}(k, \alpha)$. The considerable difference between $U_{G O M R}(\alpha)$ and $T_{\max }{ }^{F R}(\alpha)$ for $\alpha \neq 0$ can be intuitively explained by considering that, for increasing network size, FR is not able to reroute through alternative paths the traffic component which is not direct to the hot-spot node. Moreover, the asymptotic behaviour of the curves relevant to FR does not depend on $p$.

From the graph it results that also in the case of DNs and KNs the maximum throughput has the same behaviour for increasing values of $N$, i.e., it tends to 0 with decay rate $1 / \alpha N$. In all the cases, the performance of MLK and MLD is between that of FR and GOMR; in most cases, $T_{\max }^{M L K}(\alpha)$ practically coincides with $U_{G O M R}(\alpha)$. 


\section{CONCLUSIONS}

The throughput performance of Multihop Networks loaded by hot-spot traffic and operating according to minimum path length routing strategies has been analyzed. Three logical topologies based on Perfect Shuffle graphs, Kautz graphs and De Bruijn graphs have been considered.

Analytical expressions of maximum throughput values have been utilized for ShuffleNets; whereas, for Kautz and De Bruijn networks, a lower bound has been found which, in most cases, provides the exact throughput value. When this is not true, a path enumeration technique for the throughput evaluation has been used.

From the analysis it turned out that the throughput performance of the three topologies is qualitatively equivalent. More precisely, ShuffleNets and Kautz networks always slightly outperform De Bruijn graphs, while they have throughput performance practically coincident.

Generally speaking, the network performance depends fundamentally on the number of network nodes, whereas the impact of the particular choice of $p$ and $k$ is negligible for all the topologies. The only distinctive element among these topologies consists in the higher flexibility of ShuffleNets, since in these networks the hot-spot can be placed indifferently in each node.

Further work is now proceeding to find closed form expressions for the maximum throughput for Kautz and De Bruijn topologies. Moreover, impact on adaptive routing schemes on Kautz and De Bruijn networks have to be analyzed.

\section{ACKNOWLEDGEMENT}

Work carried out in the framework of the agreement between the Italian PT Administration and the Fondazione Ugo Bordoni.

\section{REFERENCES}

[1] A. S. Acampora: "A Multichannel Multihop Local Lightwave Network". Globecom'87, Tokyo (Japan), November 1987, paper 37.5.

[2] A. S. Acampora, M. J. Karol: "An Overview of Lightwave Packet Networks". IEEE Network, January 1989, pp. 29-41.

[3] M. Eisenberg, N. Mehravari: "Performance of the Multichannel Multihop Lightwave Network under non uniform traffic". IEEE JSAC, Vol. 6, $\mathrm{N}^{\circ}$ 7, August 1988.

[4] M. J. Karol, S. Z. Shaikh: "A simple adaptive routing scheme for congestion control in ShuffleNet Multihop Lightwave Networks", IEEE JSAC, Vol. 9, ํㅜ 7, September 1991, pp. 1040-1051.

[5] A. S. Acampora, S. I. A. Shah: "Multihop lightwave networks: a comparison of store-and-forward and hot-potato routing", IEEE Transactions on Communications, Vol. 40, N 6, June 1992, pp. 1082-1090.

[6] M. Kadoch, A.K. Elhakeem: "Adaptive Routing for Lightwave Shufflenet Under Unbalanced Loads". Globecom'91, San Diego (USA), November 1991, paper 52.7.

[7] F. Bernabei, L. Gratta, M. Listanti: "Throughput Analysis of Multihop ShuffleNets in a Hot-Spot Traffic Scenario: Impact of Routing Strategies". Computer Networks and ISDN Systems, Vol. 28, N6, April 1996.

[8] K. N. Sivarajan, R. Ramaswami: "Lightwave Networks Based on De Bruijn Graphs". IEEE/ACM Transactions on Networking, Vol. 2, $\mathrm{N}^{\circ} 1$, February 1994, pp. 70-79.

[9] G. Panchapakesan, A. Sengupta: "On Multihop Optical Network Topology Using Kautz Digraphs". Infocom 95, Toronto (Canada), April 1995, paper 6a.1.1, pp. 675-682. 